Draft Version June 9, 2015

Preprint typeset using $\mathrm{LAT}_{\mathrm{E}} \mathrm{X}$ style emulateapj v. 5/2/11

\title{
AN ANALYTICAL MODEL FOR GALAXY METALLICITY: WHAT DO METALLICITY RELATIONS TELL US ABOUT STAR FORMATION AND OUTFLOW?
}

\author{
Yu Lu ${ }^{1}$, Guillermo A. Blanc ${ }^{1,2}$, Andrew Benson ${ }^{1}$ \\ Draft version June 9, 2015
}

\begin{abstract}
We develop a simple analytical model that tracks galactic metallicities governed by star formation and feedback to gain insight from the observed galaxy stellar mass-metallicity relations over a large range of stellar masses and redshifts. The model reveals the following implications of star formation and feedback processes in galaxy formation. First, the observed metallicity relations provide a stringent upper limit for the averaged outflow mass-loading factors of local galaxies, which is $\sim 20$ for $M_{*} \sim 10^{9} \mathrm{M}_{\odot}$ galaxies and monotonically decreases to $\sim 1$ for $M_{*} \sim 10^{11} \mathrm{M}_{\odot}$ galaxies. Second, the inferred upper-limit for the outflow mass-loading factor sensitively depends on whether the outflow is metal-enriched with respect to the ISM metallicity. If half of the metals ejected from SNe leave the galaxy in metal-enriched winds, the outflow mass-loading factor for galaxies at any mass can barely be higher than $\sim 10$, which puts strong constraints on galaxy formation models. Third, the relatively lower stellar-phase to gas-phase metallicity ratio for lower-mass galaxies indicate that lowmass galaxies are still rapidly enriching their metallicities in recent times, while high-mass galaxies are more settled, which seems to show a downsizing effect in the metallicity evolution of galaxies. The analysis presented in the paper demonstrates the importance of accurate measurements of galaxy metallicities and the cold gas fraction of galaxies at different redshifts for constraining star formation and feedback processes, and demonstrates the power of these relations in constraining the physics of galaxy formation.
\end{abstract}

\section{INTRODUCTION}

Star formation and galactic outflows triggered by feedback from star formation are considered the most important processes in galaxy formation (Benson 2010; Mo et al. 2010). Galaxy surveys find that galaxies comprise only a small fraction of baryonic matter in the Universe (e.g. Brinchmann \& Ellis 2000; Cole et al. 2001; Dickinson et al. 2003; Bell et al. 2003). Assuming galaxies form in Cold Dark Matter (CDM) halos (Blumenthal et al. 1984), the baryon mass fraction in halos hosting a galaxy smaller than the Milky Way must decrease rapidly for decreasing halo mass in order to explain the shallow slope of the low-mass end of galaxy mass function, pointed out in pioneering works by Frenk et al. (1988); Cole (1991); White \& Frenk (1991), and elaborated by recent analysis (e.g. Papastergis et al. 2012; Behroozi et al. 2013; Moster et al. 2013; Lu et al. 2015b). Strong outflows have been proposed as the most important process responsible for keeping low-mass galaxies baryon poor (Dekel \& Silk 1986; Lacey \& Silk 1991, e.g.). Many galaxy formation models successfully reproduce the number density of low-mass galaxies by invoking strong outflows (e.g. Benson et al. 2003; Somerville et al. 2008; Guo et al.|2011). Using model inference techniques, Lu et al. (2014) and Benson (2014) found that to match the data the outflow rate needs to be more than 10 times higher than the star formation rate for low-mass galaxies. Moreover, these outflows must be sustained, or at least be recurrent, over cosmological time scales. Although strong feedback in starburst galaxies is

\footnotetext{
${ }^{1}$ The Observatories, The Carnegie Institution for Science, 813 Santa Barbara Street, Pasadena, CA 91101, USA

2 Departamento de Astronoma, Universidad de Chile, Camino el Observatorio 1515, Las Condes, Santiago, Chile
}

often observed, it is not clear if the duty cycle of the outflow is sufficiently high to be consistent with what seems to be required in the models. In spite of the success of the assumption of strong outflows in reproducing many statistical properties of the galaxy population, observational evidence for such strong outflows in local galaxies is still lacking (e.g. Bouché et al. 2012; Kacprzak et al. 2014). Therefore, it is important to seek other independent observational tests to constrain the strength of outflows.

Metals other than the primordial species can only be produced by star formation. Therefore, metallicities of the baryonic matter in different phases in a galaxy are expected to put interesting constraints on star formation and outflow. For a given galaxy, the total stellar mass provides the total metal mass budget to be distributed anywhere associated with the galaxy (including in outflows). The total amount of metal mass remaining in a galaxy, therefore, contains critical information about how outflows work. Moreover, how metals are partitioned between different phases of baryonic matter may give some insight into the nature of star formation. Using the combination of gas- and stellar-phase metallicities, one can attempt to infer the broad nature of the star formation history in a galaxy.

To extract these pieces of information from observational data, we develop an analytic model that can logically connect star formation and outflow processes with the observed metallicity relations. Using this model, we attempt to gain insight into these metallicity relations and to constrain the strength of outflow and the metallicity of past star formation. We find that with simple but plausible assumptions such a model can match the observed metallicity relations and draw inferences about star formation and outflow based on existing data.

The paper is organized as follows. In $\S 2$, we describe 
the model of the evolution of metal content of galaxies which is based on minimal assumptions. We develop two different approaches to constrain the mass-loading factors of galactic outflows and star formation in different environments using data on the mass-metallicity relations. In $\S 3$, we describe the observational data adopted in this paper. We then demonstrate in $\S \square$ how observational data on the stellar- and gas-phase metallicities constrain the outflow mass-loading factor and star formation. In $\S 5$, we discuss the conclusions and implications of these results. Throughout the paper, we assume Solar metallicity $Z_{\odot}=0.0134$ (Asplund et al. 2009) and Solar oxygen abundance $12+\log (\mathrm{O} / \mathrm{H})_{\odot}=8.69$ (Allende Prieto et al. 2001; Asplund et al. 2009). We adopt a Chabrier IMF (Chabrier 2003) for all data and modeling. We have converted all the adopted data based on these assumptions. Another important quantity we need to fix is the chemical yield, $y$, which is defined as the ratio between the mass of newly produced metals that are ejected into the interstellar gas and the mass locked in long-lived stars for a single stellar population, as originally defined by Searle \& Sargent (1972). Note that this is different from another widely adopted definition of yield in the literature, $p$, which is defined as the mass of newly produced metals per unit gas mass that is turned into stars (i.e. these two definitions differ due to the recycling of gas from short-lived stars). In this paper, we choose to use a rather high metal yield, $y=$ 0.07 , which corresponds to $p=0.038$ for the Chabrier IMF. This value is at the higher end of values predicted by nucleo-synthesis models (Woosley \& Weaver 1995; Woosley \& Heger 2002; Nomoto et al. 1997b,a). We choose to use this high yield to have a conservative estimate of the upper limit of the inferred outflow massloading factor.

\section{AN ANALYTIC MODEL FOR GALAXY METALLICITY}

The origin and implication of galaxy metallicity relations are extensively studied by many authors using analytical models (e.g. Tinslev \& Larson 1978; Matteucci \& Tornambe 1987; Köppen \& Edmunds 1999; Dalcanton 2007; Erb 2008; Davé et al. 2012; Peeples \& Somerville 2013; Pipino et al. 2014; Zahid et al. 2014), semi-analytic galaxy formation models (e.g. Cole et al. 2000; De Lucia et al. 2004; Nagashima et al. 2005; Yates et al. 2013), and hydrodynamical simulations (e.g. Finlator \& Davé 2008; Davé et al. 2011; Kobayashi et al. 2007; Wiersma et al. 2009; Ma et al. 2015). In this paper, we take the analytical approach to build a simple model that connects the buildup of metal mass in a galaxy and outflows of material from that same galaxy. In this model, star formation happens in the interstellar medium (ISM), which is continuously enriched with metals by star formation. We assume that metals are instantaneously produced by star formation, and the time delays between the nuclear synthesis of different heavy elements are ignored for simplicity (i.e. the usual "instantaneous recycling approximation"). In addition, we assume that the mixing of metal mass in the ISM is perfect. For simplicity, we further assume that the newly accreted gas does not contribute any significant metal mass.

Under these assumptions, we can write down a set of equations to follow the metal mass. The change of the metal mass in the ISM when $\mathrm{d} M_{*}$ of stellar mass is formed can be written as

$$
\mathrm{d} M_{\mathrm{z}, \mathrm{g}}=y \mathrm{~d} M_{*}-Z_{\mathrm{g}} \mathrm{d} M_{*}-\frac{\eta}{1-R} Z_{\mathrm{g}} \mathrm{d} M_{*},
$$

where $M_{z, g}$ denotes the metal mass in cold gas, $M_{*}$ denotes the mass of long-lived stars, $y$ is the chemical yield, defined as the ratio between the mass of newly produced metals and the mass locked in long-lived stars (Searle \& Sargent 1972), $Z_{\mathrm{g}}$ is the gas-phase metallicity at the time of star formation, $R$ is the mass fraction that is returned into ISM from short lived stars and stellar wind, and $\eta$ is the galactic outflow mass-loading factor, which is defined as the ratio between the outflow mass flux and the instantaneous star formation rate. The first term in the right side of the equation represents the metal mass newly formed and returned by star formation; the second term represents the metal mass locked into longlived stars; the third term represents the metal mass in the ISM that is carried away by outflows.

We note that the chemical yield $y$ and the return fraction $R$ are approximately constant parameters, which are largely determined by the stellar initial mass function (IMF) and weakly depend on metallicity (e.g. BC03 Bruzual \& Charlot 2003). For a Chabrier IMF (Chabrier 2003), $R=0.46$ is relevant to adopt for the instantaneous recycling approximation. The outflow mass-loading factor $\eta$ is a variable to be constrained in this paper. In principle, the outflow mass-loading factor $\eta$ is a variable that can change with time and from galaxy to galaxy. In this paper, we assume that $\eta$ can arbitrarily vary as a function of the present-day galaxy stellar mass, but is a constant for a given galaxy (i.e. is not a function of time). This is equivalent to assuming that the parameter $\eta$ is an effective mass-loading factor averaged over the history of the galaxy and weighted by the star formation rate, i.e. $\eta=\int_{0}^{t_{0}} \eta(t) \phi(t) \mathrm{d} t / \int_{0}^{t_{0}} \phi(t) \mathrm{d} t$, where $\phi(t)$ is the time dependent star formation rate. We note that because stellar mass can only increase for an isolated galaxy, one can use stellar mass as a clock to integrate the increase of the metal mass over time.

Using this simple model, we can derive a set of equations to describe the relationship between metallicities and parameters characterizing star formation and outflow. In the first set of derivations, we seek the relationship between the stellar-phase metallicity, gas-phase metallicity and the outflow mass-loading factor. The change of the metal mass locked into long-lived stars in a time interval is the stellar mass formed in the time interval multiplied by the instantaneous gas-phase metallicity $Z_{\mathrm{g}}$, as

$$
\mathrm{d} M_{\mathrm{z}, *}=Z_{\mathrm{g}} \mathrm{d} M_{*} .
$$

From this equation, one realizes that the term $Z_{\mathrm{g}} \mathrm{d} M_{*}$ in Equation (10) is just the metal mass that is locked into long-lived stars. The integral of Equation (11) immediately yields that

$$
M_{\mathrm{z}, \mathrm{g}}=y M_{*}-\left(1+\frac{\eta}{1-R}\right) Z_{*} M_{*},
$$

where $Z_{*}$ is the averaged stellar-phase metallicity. Adopting the definition for the gas-phase metallicity, $Z_{\mathrm{g}}=M_{\mathrm{z}, \mathrm{g}} / M_{\mathrm{g}}$, we find that the gas-phase metallicity 
is

$$
Z_{\mathrm{g}}=\frac{y}{r_{\mathrm{g}}}-\left(1+\frac{\eta}{1-R}\right) \frac{Z_{*}}{r_{\mathrm{g}}},
$$

where $r_{\mathrm{g}}$ is the gas-to-stellar mass ratio of galaxies at the present day, e.g. $r_{\mathrm{g}}=M_{\mathrm{g}} / M_{*}$. We note that how the gas-to-stellar mass ratio evolves in the past is irrelevant here, because this equation only describes the presentday metallicities of a galaxy. This equation shows that the gas-phase metallicity decreases with an increasing gas-to-stellar mass ratio, as the cold gas dilutes the metallicity of the ISM. It also shows that the gas-phase metallicity decreases with an increasing outflow massloading factor, as outflow expels metal-enriched gas out of galaxies. We can rewrite the equation as follows to determine the mass-loading factor using metallicities,

$$
\eta=(1-R)\left[\frac{y}{Z_{*}}-\frac{Z_{\mathrm{g}}}{Z_{*}} r_{\mathrm{g}}-1\right] .
$$

This equation shows that, for a given galaxy, higher metallicities and higher cold gas-stellar mass ratio indicate a lower outflow mass-loading factor. This is easy to understand because strong outflow will reduce the metal content and gas content of the galaxy.

In the second set of derivations, we adopt the gas-phase metallicity at different redshifts as data constraints. To do so, we need to assume how the cold gas metallicity $Z_{\mathrm{g}}$ evolves with stellar mass growth. Here we first adopt a simple assumption that the gas-phase metallicity follows a power-law trajectory in the $Z_{\mathrm{g}}-M_{*}$ plane as the galaxy evolves, e.g.

$$
Z_{\mathrm{g}}(t)=Z_{\mathrm{g}}\left(\frac{M_{*}(t)}{M_{*}}\right)^{\mu},
$$

in which the power index, $\mu$, is assumed to vary as a function of galaxy stellar mass, and is to be determined. The variation of $\mu$ reflects different ways in which galaxies can evolve their metallicities as their stellar masses increase. For example, inflow can decrease metallicity, and outflow can increase or decrease the metallicity depending on how metals are loaded in the wind. The assumption of power-law trajectories allows galaxies with different masses to evolve along different paths. Based on this simple assumption, by integrating Eq1 we find that

$$
M_{\mathrm{z}, \mathrm{g}}=y M_{*}-\left(1+\frac{\eta}{1-R}\right) \frac{1}{\mu+1} Z_{\mathrm{g}} M_{*} .
$$

Applying the definition of the gas-phase metallicity, we can rewrite the equation to be an expression for the massloading factor,

$$
\eta=(1-R)\left[(\mu+1)\left(\frac{y}{Z_{\mathrm{g}}}-r_{\mathrm{g}}\right)-1\right] .
$$

This equation shows that we can also constrain the averaged mass-loading factor $\eta$, when the trajectory of a galaxy in the $Z_{\mathrm{g}}-M_{*}$ plane is determined.

In the above derivations, we have assumed that all the newly produced metals are returned into the ISM, so that the yield affecting the chemical evolution of a galaxy equals to the intrinsic nucleo-synthesis yield. Because SN ejecta can preferentially transport over-enriched (relative to the mean ISM) gas out of the potential well of a galaxy as shown in hydro-dynamical simulations (e.g. Creasev et al. 2015; (Melioli et al. 2015), it is reasonable to assume that the "retained" yield, $\tilde{y}$, is less than the intrinsic yield, $y$. In the following analysis, we vary the retained yield to explore this effect.

\section{DATA}

The derivations presented in the last section demonstrate that one can draw inferences about star formation and feedback when certain observational data are given. Based on our derivations, these observational data include the stellar-phase metallicity as a function of galaxy stellar mass, the gas-phase metallicity as a function of galaxy stellar mass of local galaxies, and of galaxies at different redshifts. To demonstrate the constraining power of these observational relations based on the analytic derivations shown in the previous section, we choose to use the mean relation of each of observational results. For the stellar-phase metallicity, we adopt the observational results of Gallazzi et al. (2005) and Kirby et al. (2013) to cover a wide range of stellar masses between $\sim 10^{7} \mathrm{M}_{\odot}$ and $10^{11} \mathrm{M}_{\odot}$. As shown by Kirby et al. (2013), these two pieces of data join with each other remarkably well if the relation determined by the Kirby data is extrapolated to high stellar masses. In the analysis of this paper, we adopt a function that follows the two data sets and smoothly joins them as shown in Fig. 11. We note that because the uncertainty in the stellar metallicities at the low mass end of the Gallazzi et al. (2005) relation is large, the small deviation between their mean relation and our adopted function is ignored in this paper. In addition, there are two biases in the stellar-phase metallicity. First, the observed metallicity is weighted by young and luminous stars, which tend to be metal rich. Second, the stellarphase metallicity measurements are mainly sensitive to iron abundances rather than the $\alpha$ elements, which are the species measured in the gas-phase metallicity measurements. Following Peeples et al. (2014), we have corrected these biases using their Eq.(7) and Eq.(A1), taking into account the different solar metallicity adopted in the reference paper. For the gas-phase metallicity relations, there are a larger number of results published in the literature. While the observed gas-phase metallicity relations have small scatter at a given redshift (typically $0.1 \mathrm{dex}$ ), they have substantial differences in their normalizations and shapes owing to large uncertainties in the calibration of the metallicity measurements (Kewley \& Ellison 2008). To take these uncertainties into account in our inferences, we adopt multiple observational results. For the gas-phase metallicity-stellar mass relation of local star forming galaxies, we adopt the results of Tremonti et al. (2004); Maiolino et al. (2008); Zahid et al. (2013) and Andrews \& Martini (2013) for galaxies with stellar mass in a range between $\sim 10^{9} \mathrm{M}_{\odot}$ and $10^{11} \mathrm{M}_{\odot}$, and Lee et al. (2006) for lower mass galaxies in the range between $\sim 10^{7} \mathrm{M}$ and $10^{9} \mathrm{M}_{\odot}$. For higher redshifts, we adopt the metallicity-stellar mass relation at $z \sim 2.2$ compiled by Maiolino et al. (2008) and Zahid et al. (2013), respectively. In addition, we also use the gas-phase metallicity measurements of Henry et al. (2013a, b) for a lower mass range between $10^{8} \mathrm{M}_{\odot}$ and $10^{10} \mathrm{M}_{\odot}$ as a complement. To infer the mass-loading factor with the approaches demon- 
strated in the previous section, one also needs to know the gas-to-stellar mass ratio of galaxies. We adopt a fitting formula for the mean relation of star forming galaxies compiled by Peeples et al. (2014), namely

$$
\log r_{\mathrm{g}}=-0.48 \log \left(\frac{M_{*}}{\mathrm{M}_{\odot}}\right)+4.39 .
$$

As shown by the authors, this fitting formula captures the mean relation between the gas mass ratio and galaxy stellar mass well and agrees very well with many observational estimates (e.g. McGaugh 2005, 2012; Leroy et al. 2008; Papastergis et al. 2012). Unlike the metallicitystellar mass relations, the cold-gas to stellar mass ratio has a significantly large scatter, $\sim 0.5$ dex. We add this scatter into the gas mass ratio as upper and lower bounds with $\Delta \log r_{\mathrm{g}}=0.5$ to demonstrate how this scatter propagates into our results. For all the data sets, we have corrected the stellar mass by assuming a Chabrier (2003) IMF.

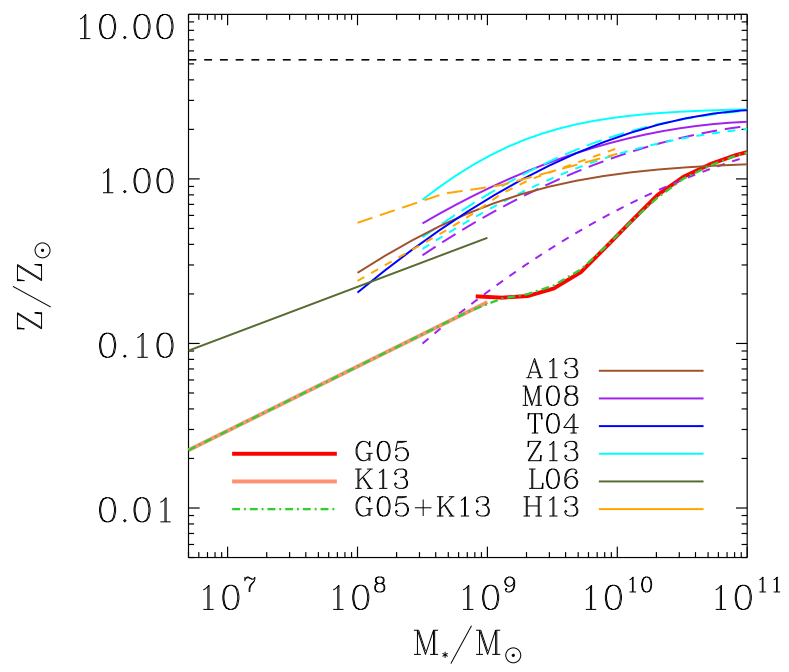

FIG. 1.- Observational results of metallicity as a function of galaxy stellar mass. The stellar-phase metallicity relations are from Gallazzi et al. (2005) and Kirby et al. (2013), denoted by the solid lines of G05 and K13, respectively. The green dashdotted line shows a smooth function joining both of the data sets. The local galaxy gas-phase metallicity relations are from Tremonti et al. (2004), Maiolino et al. (2008), Zahid et al. (2013), Andrews \& Martini (2013), and Lee et al. (2006), which are denoted by colored solid lines of T04, M08, Z13, A13, and L06, respectively. The gas-phase metallicity relations at higher redshifts of Maiolino et al. (2008) and Zahid et al. (2013) are shown by longdashed lines for $z \sim 0.7$ and short-dashed lines for $z \sim 2.2$. The gas-phase metallicity relations of Henrv et al. (2013a) for $z \sim 0.6$ and Henry et al. (2013b) for $z \sim 1.8$, denoted by H13, are shown by long-dashed and short-dashed orange lines. The horizontal short dashed line denotes the intrinsic yield $y$ assumed in the paper.

\section{RESULTS}

\subsection{Outflow mass-loading factor Inferred from local metallicity relations}

As we demonstrated in $\$ 2$ without assuming any particular star formation history or metallicity enrichment history, our simple model can match the stellar-phase and gas-phase metallicity relations at a given redshift and

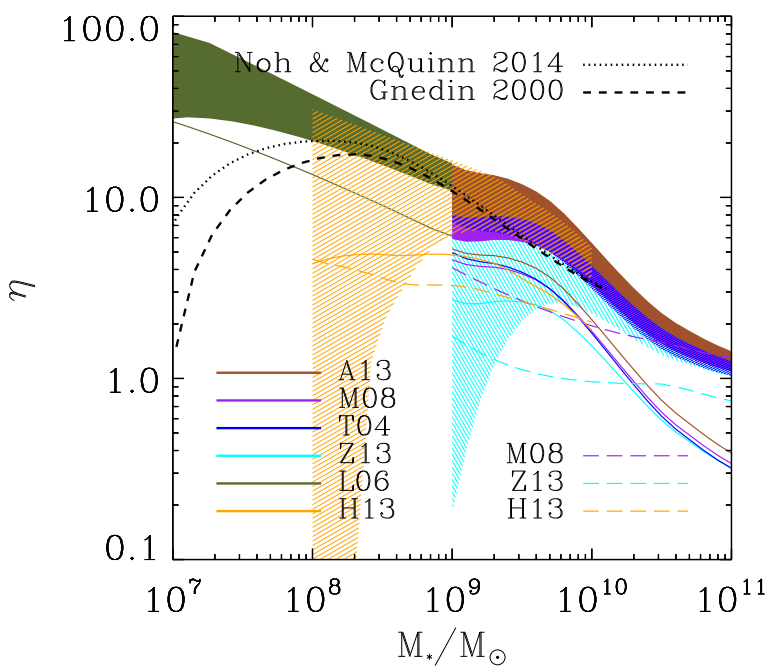

FIG. 2.- The constrained outflow mass-loading factor $\eta$ derived from different combinations of observational data. The shaded regions and the solid lines are the results inferred from the stellarphase metallicity and the gas-phase metallicity of local galaxies. The shaded regions show $\eta$ obtained by assuming that all produced metal mass is mixed into the ISM but varying the cold gas to stellar mass ratio. The upper and lower bounds encompass the variations resulted from the \pm 0.5 dex scatter of the cold gas mass ratio for given stellar mass. The solid lines show the results assuming half of the produced metal is directly expelled from galaxies, i.e. $\tilde{y}=0.5 y$ with the fiducial cold gas mass ratio. The dashed lines denote the results inferred from the gas-phase metallicity relation at multiple redshifts (see 4.2). Different colors denote different data sources. The brown line represents the result using Andrews \& Martini (2013) data. The purple lines denote the results using Maiolino et al. (2008) data. The blue line shows the result using Tremonti et al. (2004) data. The cyan lines show the results using Zahid et al. (2013) data. The orange line shows the results using Henry et al. (2013a b) data. The green line shows the result using Lee et al. (2006) data. The black dotted and dashed lines denote the upper limit estimates of the mass-loading factor allowed by two different re-ionization models.

can constrain the mass-loading factor. In this subsection, we adopt the combined the stellar-phase metallicity as a function of stellar mass and the gas-phase metallicity results of Tremonti et al. (2004); Maiolino et al. (2008); Zahid et al. (2013); Andrews \& Martini (2013), and Lee et al. (2006) of local galaxies to infer the outflow mass-loading factor, $\eta$, as a function of galaxy stellar mass. We also include the gas-phase metallicity relation of Henry et al. (2013a) at $z=0.6$ in this analysis. We choose each of the gas-phase metallicity relation and combine it with the stellar-phase metallicity relation and the gas-to-stellar mass ratio to feed into Equation (5). At first, we assume all of the metals produced are retained in the galaxy and mixed into ISM before they can be expelled by outflows, i.e $\tilde{y}=y$. In other words, outflows have the same metallicity as the ISM with no further metallicity enhancement. We vary the normalization of the gas-to-stellar mass ratio, $r_{\mathrm{g}}$, up and down by 0.5 dex to encompass the random variation of the gas mass for given stellar mass. The resulting massloading factor as a function of galaxy stellar mass for each data set is shown by the shaded bands in Fig. 2 For the all results, the general trend is that the massloading factor is higher for low-mass galaxies and decreases rapidly for higher stellar masses. As one can see, 
the inferred $\eta-M_{*}$ relations by using different gas-phase metallicity relations are very similar to each other at the high-mass end, where the inferred mass-loading factor is less than 2. Another interesting feature is that the inferred mass-loading factor tends to flatten out at the very high mass end. The reason for this is that mergers become increasingly important for higher mass galaxies, and the mass-loading factor we constrain is considered to be an average over all progenitors, which have relatively lower masses. In the lower mass regime, the scatter in the gas mass ratio produces increasingly larger variations in the inferred outflow mass-loading factor. Nevertheless, the upper bound for $\eta$ is well defined. For galaxies $M_{*}=10^{9} \mathrm{M}_{\odot}$, the upper-bound mass-loading factor inferred from all the data combinations is less than 20 . For the same stellar mass, galaxies with higher gas fraction may have significantly lower $\eta$. In addition, different gas-phase metallicity results have different level of sensitivity to the variation of the gas mass ratio. A higher gas-phase metallicity not only suggests a systematically lower mass-loading factor, but also a more sensitive dependence on the gas mass ratio. Second, we assume that SN ejecta carry away half of the produced metal mass without mixing it into ISM. In this situation, $\tilde{y}=0.5 y$, and we show the inferred $\eta$ with the median gas mass ratio by solid lines in Fig.2. When a fraction of metal leaks from galaxies without mixing into ISM, the observed metallicity relations yield a much lower $\eta$. At the high-mass end, all the data combinations suggest that the mass-loading factor is below 1 . At $M_{*}=10^{9} \mathrm{M}_{\odot}$, the mass-loading factor is below 10, and it increases with decreasing stellar mass. At the low-mass end of the mass range $\left(\sim 10^{7} \mathrm{M}_{\odot}\right)$, the mass-loading factor is about 20 . It is worth noting that we chose to use a rather high metal yield, $y=0.07$, which is about five times of the Solar metallicity, and at the high end of usual values predicted by neucleo-synthesis models (Woosley \& Weaver 1995; Nomoto et al. 1997b, a). We chose this high yield parameter to demonstrate an upper limit for the massloading factor. If a lower metal yield is chosen instead, the inferred mass-loading factor decreases.

For $10^{7} \mathrm{M}_{\odot}$ galaxies, the mass-loading factor inferred by the Lee et al. (2006) gas-phase metallicity and the Kirby et al. (2013) stellar-phase metallicity relations can be as high as $\sim 20$. We note, however, that low-mass galaxies are expected to be hosted by low-mass halos, and re-ionization would prevent a large fraction of baryonic mass from collapsing into such low-mass halos in the first place, so that galaxies forming in those halos would never have contained as much baryonic mass as these large mass-loading factors implied, unless the outflow materials are rapidly reaccreted back into the galaxy. We note that $\eta$ is the averaged net mass-loading factor, while the instantaneous mass loading in individual starburst galaxies may be higher if there is significant baryon mass recycled from early times. Under the assumption that reaccretion is not important, the total baryonic mass in stars, cold gas, and ejected by outflow should be lower than the total baryonic mass that can collapse into the host halo, i.e.

$$
M_{*}+M_{*} r_{\mathrm{g}}+\eta \frac{M_{*}}{1-R} \leq f_{\mathrm{b}} f_{\text {reion }}\left(M_{\mathrm{vir}}, z\right) M_{\mathrm{vir}}
$$

where $f_{\mathrm{b}}=0.17$ is the cosmic baryon fraction, and $f_{\text {reion }}$ is the fraction of baryon mass that can collapse into a halo with virial mass $M_{\text {vir }}$ at redshift $z$ due to reionization. This inequality yields that

$$
\eta \leq(1-R)\left[f_{\mathrm{b}} f_{\text {reion }} \frac{M_{\mathrm{vir}}}{M_{*}}-r_{\mathrm{g}}-1\right] .
$$

To estimate the limit for the mass-loading factor, we adopt a re-ionization model by Gnedin (2000) with a fitting formula proposed by Kravtsov et al. (2004) and a recent model by Noh \& McQuinn (2014) to compute the total baryon mass fraction as a function of halo mass at $z=0$. We then adopt the abundance matching model of Behroozi et al. (2013) and extrapolate it to the low-mass end $\left(M_{*} \sim 10^{7} \mathrm{M}_{\odot}\right)$ to determine the stellar mass-halo mass relation. Using these and Equation (11), we can derive an upper limit for the mass-loading factor allowed by re-ionization. The thick dashed line and dotted line in Fig 2 show the upper limits set by the Gnedin model and the Noh \& McQuinn model, respectively. In both cases, the limiting outflow mass-loading factor drops sharply when galaxy stellar mass goes below $\sim 10^{8} \mathrm{M}_{\odot}$. Below this mass scale, re-ionization prevents baryons from collapsing into the low-mass halos hosting these galaxies. These low-mass galaxies should therefore never have contained as many baryons as is implied by their inferred mass-loading factors given their existing stellar mass and cold gas mass. To reconcile the observed metallicities and cold baryon masses of low-mass galaxies, a more plausible model seems to require metal-enriched outflows, which carry away a large fraction of metals directly from SN ejecta without mixing much metals win ISM. In this scenario, the retained metal yield can be lower than we used here, yielding lower mass-loading factors.

\subsection{Outflow mass-loading factor inferred from gas-phase metallicity relations at different redshifts}

We now use the gas-phase metallicity relation at multiple redshifts to carry out the inference described in 42 We adopt the gas-phase metallicity-stellar mass relation compiled by Maiolino et al. (2008) for galaxies at two different redshifts $z=0.07$ and $z=2.2$, the relation compiled by Zahid et al. (2013) at $z=0.08$ and $z=2.3$, and the relation measured by Henry et al. (2013a, b) at $z=0.6-0.7$ and $z=1.3-2.3$ for lower stellar masses $\left(M_{*}=10^{8}-10^{10} \mathrm{M}_{\odot}\right)$. As we described in 92 we use this data to determine the trajectories along which galaxies evolve in the $Z-M_{*}$ diagram. For the simple case we demonstrated in 82 , we essentially need to determine the logarithmic slope, $\mu$, as a function of galaxy stellar mass using the observed gas-phase metallicity relations at two separate redshifts. One way to determine $\mu\left(M_{*}\right)$ is to use a realistic star formation history of a galaxy with a stellar mass $M_{*}$ at a lower redshift to determine the stellar mass of its typical progenitor at a higher redshift, and then determine where the progenitor galaxy is in the $Z_{\mathrm{g}}-M_{*}$ diagram at the higher redshift by using the gasphase metallicity-stellar mass ration at that redshift. We adopt a fitting model for the star formation histories of star forming galaxies proposed by Leitner (2012),

$$
\phi\left(M_{*}, z\right)=A_{0}\left(\frac{M_{*}}{10^{11} \mathrm{M}_{\odot}}\right)^{\beta+1}(1+z)^{\alpha},
$$




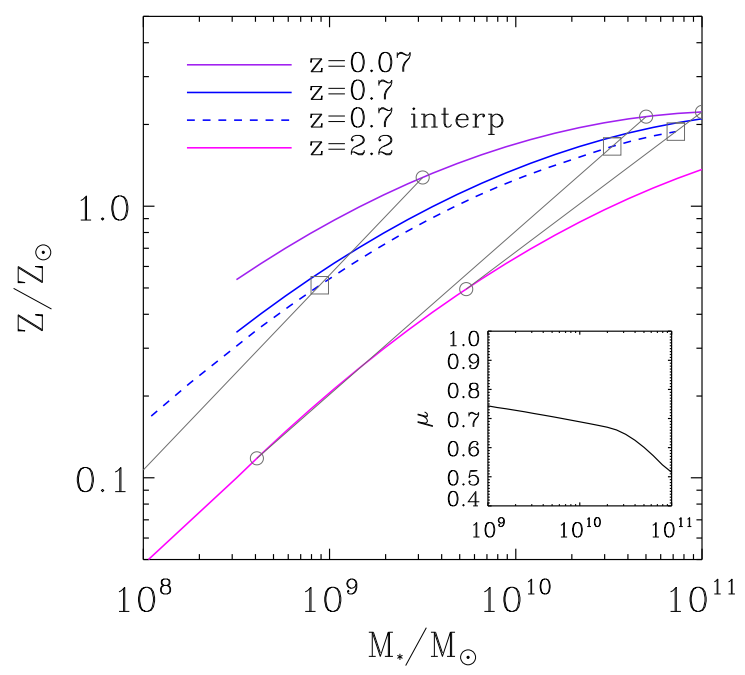

FIG. 3.- The grey lines show three examples of the power-law trajectories of galaxies in the $Z_{\mathrm{g}}-M_{*}$ diagram. These trajectories are determined by assuming star formation histories parameterized by Equation (12) and the gas-phase metallicity relations at $z=0.07$ and $z=2.2$ from Maiolino et al. (2008). The blue dashed line shows the predicted gas-phase metallicity as a function of stellar mass at $z=0.7$ using the determined trajectories. The solid lines are the observational results of Maiolino et al. (2008) at different redshifts as noted in the legend. The inserted diagram shows the inferred $\mu$ (the power index for the metallicity-stellar mass trajectory) as a function of final stellar mass in units of $\mathrm{M}_{\odot}$.

where parameters $A_{0}=3.24 \mathrm{M}_{\odot} \mathrm{yr}^{-1}, \alpha=3.45$, and $\beta=-0.35$ are determined by matching the abundances of star forming galaxies out to high redshift $(z \sim 4)$. Using this model, we can integrate the star formation rate history to predict the stellar mass at any given redshift for a galaxy with a given final stellar mass. For simplicity, we again assume instantaneous recycling when integrating SFR histories. For more accurate models taking into account time-dependent recycling, readers are referred to Leitner \& Kravtsov (2011), Leitner (2012) and Lu et al. (2015a). Assuming a galaxy evolves along a power-law trajectory on the gas-phase metallicity-stellar mass plain between two different redshifts, we can find where the trajectory determined by the star formation history intersects with the gas-phase metallicity-stellar mass relations at the different redshifts. Using the two intersection points in the $Z_{\mathrm{g}}-M_{*}$ diagram, we can determine the logarithmic slope as $\mu=\left(\log Z_{\mathrm{g}}^{0}-\right.$ $\left.\log Z_{\mathrm{g}}^{1}\right) /\left(\log M_{*}^{0}-\log M_{*}^{1}\right)$, where the superscripts " 0 " and " 1 " denote quantities at two different redshifts of a galaxy. Fig 3 shows an example of how the $\mu$ parameter is determined as a function of stellar mass using the Maiolino et al. (2008) data. In the figure, we show three trajectories with different final galaxy masses in grey lines and over-plot them with the gas-phase metallicity relation of Maiolino et al. (2008) at $z=0.07,0.7$, and 2.2. For each given final stellar mass at $z=0.07$, we compute the stellar mass at $z=2.2$ using the star formation history of Eq12. We read the gas-phase metallicity of the galaxy with the stellar mass from the gasphase metallicity relation at the corresponding redshift. The open circles along a grey line are the positions of the galaxy at the two redshifts, $z=0.07$ and 2.2 . The grey lines connecting the two circles are the power-law trajectories of the three example galaxy masses. The grey squares between the two circles on each trajectory mark the predicted gas-phase metallicity-stellar mass relation at $z=0.7$. We predict those quantities at the redshift by interpolating along the star formation history and the power-law $Z-M_{*}$ trajectory. Connecting these predictions, the dashed line shows the predicted gas-phase metallicity-stellar mass relation at $z=0.7$. As one can see, the interpreted gas-phase metallicity relation at $z=0.7$ agrees with the observational result at the same redshift remarkably well, which demonstrates that the power-law trajectory sufficiently well captures the evolution of a galaxy in the $Z_{\mathrm{g}}-M_{*}$ plane. In the inserted panel in Fig 3. we show the determined $\mu$ as a function of final galaxy stellar mass. The resulting $\mu$ slowly decreases from 0.73 at $M_{*}=10^{9} \mathrm{M}_{\odot}$ to 0.51 at $M_{*}=10^{11} \mathrm{M}_{\odot}$. Peeples \& Somerville (2013) have performed more detailed inference on the gas-phase metallicity trajectories by assuming a fundamental metallicitystellar mass-SFR relation (Mannucci et al. 2010) without enforcing the trajectories to follow a power-law form. From Figure 1 of Peeples \& Somerville (2013), one can find that their trajectories are nearly power-law over a large range of redshifts, which in turn supports our simple assumption of power-law trajectories made in Equation (6).

Combining the determined $\mu\left(M_{*}\right)$ and the data and inserting these terms into Equation (8), we can again compute the mass-loading factor as a function of stellar mass. The results are shown as long dashed lines in Fig.2 As one can see, the results obtained from this approach are qualitatively similar to those inferred without assuming a star formation history in 4.1 The constrained mass-loading factor decreases with increasing stellar mass. Quantitatively, using the same yield, $\tilde{y}=y=0.07$, the mass-loading loading factors inferred in this approach are a factor of 2-3 lower than those inferred in 4.1 . The difference may be due to the calibration of different data sets or inconsistency between the empirical star formation history and the real star formation history of the star-forming galaxies included in the gas-phase metallicity measurements.

\subsection{Metallicity history}

The metallicity of a star approximately tells us the metallicity of the ISM at an early time when the star formed, while the gas-phase metallicity tells us the current metal content of the ISM. Using these two pieces of information, one can gain some insight into the metal enrichment history of galaxies. The left panel of Figure 4 shows the ratio between the stellar-phase metallicity and the gas-phase metallicity as a function of stellar mass of local galaxies. For the gas-phase metallicity relations, we adopt the results of Andrews \& Martini (2013), Maiolino et al. (2008), Tremonti et al. (2004), Zahid et al. (2013), and Lee et al. (2006) for local galaxies. For the stellar-phase metallicity, we use the Gallazzi et al. (2005) result and Kirby et al. (2013) result for relevant stellar mass range. Although these data sets have different amplitudes due to different calibrations, they show a systematic trend that low-mass galaxies $\left(M_{*}<10^{10} \mathrm{M}_{\odot}\right)$ have a relatively lower stellar-phase 

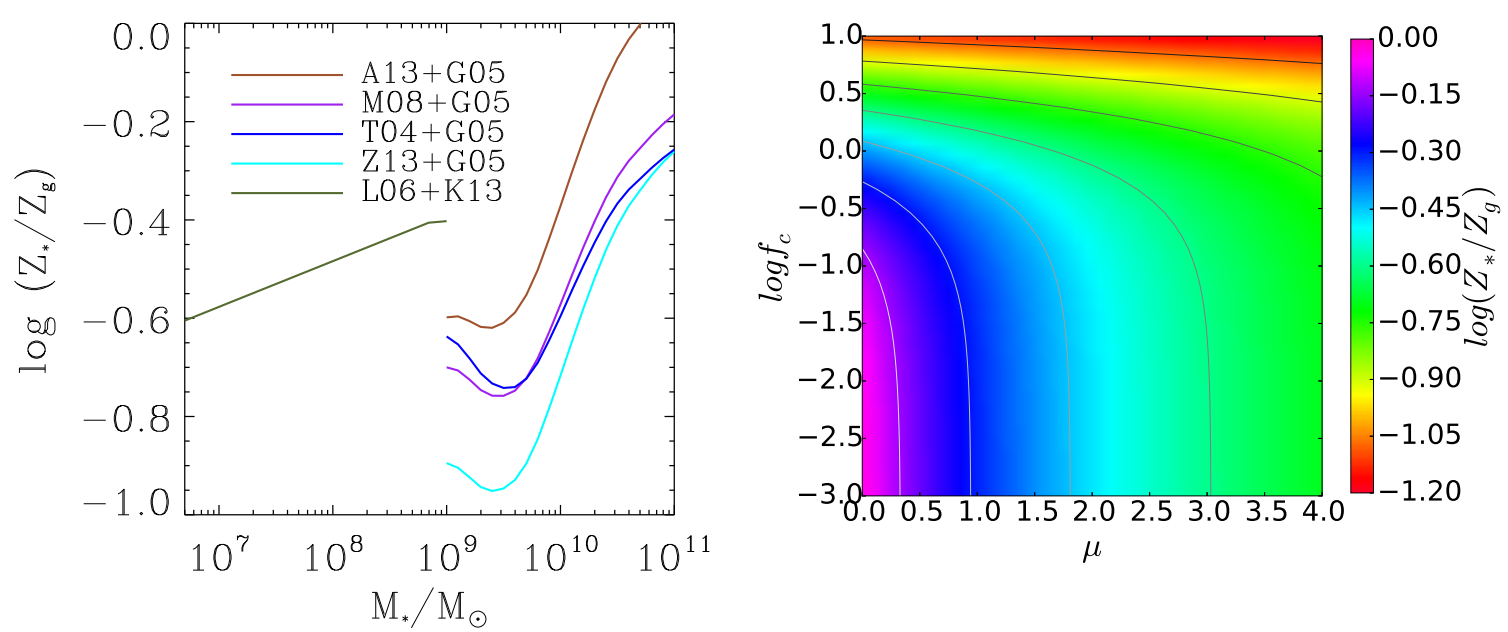

FIG. 4. - The left panel shows the stellar-phase metallicity to gas-phase metallicity ratio as a function of stellar mass. The local galaxy gas-phase metallicity relations are adopted from Tremonti et al. (2004), (Maiolino et al. (2008), Zahid et al. (2013), Andrews \& Martini (2013), and Lee et al. (2006), which are denoted by colored solid lines of T04, M08, Z13, A13, and L06, respectively. Either the Gallazzi et al. result (G05) or the Kirby et al. result (K13) for the stellar-phase metallicity is adopted for the relevant stellar mass range. The right panel shows the stellar-phase metallicity to gas-phase metallicity ratio as a function of parameter $\mu$ and $f_{\mathrm{c}}$ in Equation (15).

metallicity to gas-phase metallicity ratio than the highmass ones. This trend indicates that galaxies with different masses follow different ways of metal enrichment.

Assuming that the gas-phase metallicity trajectory in the $Z_{\mathrm{g}}-M_{*}$ plane follows a power-law function, we find that the ratio of the stellar phase metallicity and the gas-phase metallicity is

$$
\frac{Z_{*}}{Z_{\mathrm{g}}}=\frac{1}{\mu+1},
$$

where $\mu$ is the power-low index of the trajectory. What this equation tells us is that a lower stellar-phase metallicity to gas-phase metallicity ratio corresponds to a steeper gas-phase metallicity trajectory (larger $\mu$ ). The left panel of Figure 4 shows that high-mass galaxies have higher $Z_{*} / Z_{\mathrm{g}}$, which means they need to have lower $\mu$ than low-mass galaxies. This is consistent with the $\mu-M_{*}$ relation we derived from the gas-phase metallicity relation at different redshifts in 4.2 (see the inserted panel of Fig.(3). This suggests that high-mass galaxies seem to maintain a slow increase of metallicity for a long time in the past, while low-mass galaxies have rapidly enriched their metallicities in recent times.

We can also use a more sophisticated model to describe the trajectory, as the power-law model might be too simple to capture the entire evolution, especially at early times. We choose the following model using a power-law to describe the late time evolution, and an exponential term to capture the possible rapid increase of metallicity following a starburst phase at the early time, i.e.

$$
Z_{\mathrm{g}}(t)=\frac{Z_{\mathrm{g}, 0}}{K}\left[\frac{M_{*}(t)}{M_{\mathrm{c}}}\right]^{\mu} \exp \left[-\frac{M_{\mathrm{c}}}{M_{*}(t)}\right]
$$

where $K=\left(\frac{M_{*, 0}}{M_{\mathrm{c}}}\right)^{\mu} \exp \left(-\frac{M_{\mathrm{c}}}{M_{*, 0}}\right)$ is a normalization factor, $M_{*, 0}$ and $Z_{\mathrm{g}, 0}$ are the final stellar mass and gas-phase metallicity, $\mu$ parameter is the power-law slope of the trajectory of $Z_{\mathrm{g}}$ when $M_{*}>M_{\mathrm{c}}$, and $M_{\mathrm{c}}$ is a characteristic mass scale at which the $Z_{\mathrm{g}}$ trajectory switches from an exponential function to a power-law. Below $M_{\mathrm{c}}$, the gas-phase metallicity increases exponentially with mass. This model captures the idea that feedback might be powerful enough to expel all produced metals at the early epoch of galaxy formation, and it becomes less powerful at late time when the galaxy mass is high so that metals can be retained.

Using this model for the trajectory, we find that

$$
\frac{Z_{*}}{Z_{\mathrm{g}}}=f_{\mathrm{c}} \exp \left(f_{\mathrm{c}}\right) \Gamma\left(-\mu-1, f_{\mathrm{c}}\right),
$$

where $f_{\mathrm{c}} \equiv M_{\mathrm{c}} / M_{*, 0}$, and $\Gamma(s, x)$ is the upper incomplete gamma function: $\Gamma(s, x)=\int_{x}^{\infty} t^{s-1} e^{-t} \mathrm{~d} t$. We show the two-dimensional function in the right panel of Figure 4 The contours in the figure show the combinations of $f_{\mathrm{c}}$ and $\mu$ that give rise to a constant $Z_{*} / Z_{\mathrm{g}}$. As one can see, when $Z_{*} / Z_{\mathrm{g}} \sim 1$, the gas-phase metallicity track is required to be flat ( $\mu \sim 0$ and $f_{\mathrm{c}}$ is very small). When $Z_{*} / Z_{\mathrm{g}}$ becomes smaller, the gas-phase metallicity trajectory has to be a steep function of stellar mass, either with a large power-law slope $\mu$ or a higher $f_{\mathrm{c}}$. To result in this type of metallicity trajectory, a large fraction of stellar mass needs to form in an early starburst with very low metallicity, and to retain metals in the ISM to rapidly enrich the gas-phase metallicity at late time.

This inference has two implications. First, the low $Z_{*} / Z_{\mathrm{g}}$ ratio of low-mass galaxies seems to indicate that a relatively large fraction of stellar mass of these galaxies formed in early starbursts when the ISM metallicity is still low. Second, low-mass galaxies, as opposed to high-mass galaxies, are more rapidly enriching their metallicity, indicating a downsizing effect that low-mass galaxies are less evolved than the high-mass ones also in metallicity.

\section{DISCUSSION}


In this paper, we have introduced a simple analytic model to follow the metallicities of galaxies. When the model is matched to the gas-phase and stellar-phase metallicities as functions of stellar mass of galaxies at different redshifts, these observational data in turn can constrain star formation and feedback.

With minimum assumptions about how metals produced by star formation are mixed into the ISM and how outflow can affect the baryonic content of galaxies, we demonstrate that metallicities of galaxies provide useful constraints on the strength of galaxy outflows. Using the model, we have derived two approaches to constrain the outflow mass-loading factor as a function of galaxy stellar mass. In the first approach, we find the stellarphase metallicity, gas-phase metallicity and the cold-gasto-stellar mass ratio as functions of galaxy stellar mass at a given redshift can already provide strong constraints on the upper limit for the mass-loading factor. The fundamental reason that the combination of these data can constrain outflow is because the gas-phase metallicity represents the metal content of galaxies at a fixed time, and the stellar-phase metallicity represent a timeaveraged value for the metal content of galaxies. The combination of these two quantities provide constraints on how the metal content changes over time when galaxies build up their stellar mass. The metal mass that is not found in galaxies should have been carried away by SN ejecta or galactic outflow. The second approach relies on the gas-phase metallicity relation at different redshifts. Once the manner in which galaxies change their metallicity as they increase their stellar mass is determined, the metallicity relations at different epochs can also constrain the strength of outflow.

Combining multiple observational measurements for the stellar mass-metallicity relations, we have derived the outflow mass-loading factor based on the simple model. The results show that, in spite of the large uncertainties in the metallicity measurements, the inferred outflow mass-loading factors have a well defined upper limit. Even with generous (high) metal yield and assuming all produced metals are mixed into the ISM, the inferred mass-loading factor cannot be higher than 20 for $10^{9} \mathrm{M}_{\odot}$ galaxies and it drops quickly for higher galaxy masses. On the very low mass end, where re-ionization is effective to block a significant fraction of baryons from collapsing into the galaxies, strong outflow is not a plausible explanation for the cold baryon mass fraction and the metallicity relations. To explain the data, we find that a significant fraction of produced metals need to be ejected directly out of the galaxies with SN ejecta without mixing in the ISM, which is consistent with the results of Dalcanton (2007), who found that metal- enriched outflow is the only viable mechanism to reproduce the low retained yields of gas-rich low-mass galaxies. Observations of metal content of winds from dwarf starburst also suggest that almost all the metals produced in starbursts are ejected directly out of the galaxies (e.g. Martin et al. 2002), providing observational support of metal-enriched ejection. If a moderate fraction (half) of metal mass is involved in the metal-enriched ejection, the mass-loading factor is expected to be at most only a few $(\eta<8)$. This low mass-loading factor conflicts with many galaxy formation models, which require much higher mass-loading factors, especially for low-mass galaxies $\left(M_{*} \leq 10^{10} \mathrm{M}_{\odot}\right)$, to explain low-mass end of the stellar mass function (Lu et al. 2014; Benson 2014). In many successful galaxy formation models, strong outflow is needed because baryons other than observed stars and cold gas cannot stay in the halos without forming stars. The only way to keep low-mass halos from having a too high baryon mass fraction is to eject a large amount of baryonic mass out of the halo. What the metallicity relations seem to show in this paper, however, is that when galaxies increase their stellar mass (and simultaneously produce metals), the metal mass in galaxies increases substantially. The increase in the metal mass suggests that there should not be very strong outflow to take away metals.

The data show that low-mass galaxies tend to have a lower stellar-phase to gas-phase metallicity ratio. Using simple analytic models, we conclude that this trend suggests that low-mass galaxies tend to increase their gasphase metallicity rapidly in late times, while high-mass galaxies have gone through the rapid metal enrichment phase in early times, which indicates an imprint of the downsizing effect in the metallicity evolution of galaxies. The analysis also suggests that before the rapid enrichment epoch, a fraction of stellar mass is formed at early times when the gas-phase metallicity was low. This is consistent with recent observational results that local dwarf galaxies have more than half of their stellar mass form in early phase prior to $z=2$ (Weisz et al. 2014).

Finally, we stress the importance of the accuracy of the metallicity relations and cold gas mass fraction in observational measurements. We have adopted simplified version of existing observational results to demonstrate the constraining power of the data. Better observational determination of these scaling relations will definitely improve the constraints when more sophisticated model inferences, such as Bayesian model inference (e.g. Lu et al. 2011), are adopted.

\section{ACKNOWLEDGEMENTS}

We thank Andrew McWilliam, Houjun Mo, Josh Simon for useful discussions.

\section{REFERENCES}

Allende Prieto, C., Lambert, D. L., \& Asplund, M. 2001, ApJ, $556, \mathrm{~L} 63$

Andrews, B. H., \& Martini, P. 2013, ApJ, 765, 140

Asplund, M., Grevesse, N., Sauval, A. J., \& Scott, P. 2009, ARA\&A, 47, 481

Behroozi, P. S., Wechsler, R. H., \& Conroy, C. 2013, ApJ, 770, 57

Bell, E. F., McIntosh, D. H., Katz, N., \& Weinberg, M. D. 2003, ApJ, 585, L117

Benson, A. J. 2010, Phys. Rep., 495, 33
-. 2014, MNRAS, 444, 2599

Benson, A. J., Frenk, C. S., Baugh, C. M., Cole, S., \& Lacey, C. G. 2003, MNRAS, 343, 679

Blumenthal, G. R., Faber, S. M., Primack, J. R., \& Rees, M. J. 1984, Nature, 311, 517

Bouché, N., Hohensee, W., Vargas, R., et al. 2012, MNRAS, 426, 801

Brinchmann, J., \& Ellis, R. S. 2000, ApJ, 536, L77

Bruzual, G., \& Charlot, S. 2003, MNRAS, 344, 1000 
Chabrier, G. 2003, PASP, 115, 763

Cole, S. 1991, ApJ, 367, 45

Cole, S., Lacey, C. G., Baugh, C. M., \& Frenk, C. S. 2000, MNRAS, 319, 168

Cole, S., Norberg, P., Baugh, C. M., et al. 2001, MNRAS, 326, 255

Creasey, P., Theuns, T., \& Bower, R. G. 2015, MNRAS, 446, 2125

Dalcanton, J. J. 2007, ApJ, 658, 941

Davé, R., Finlator, K., \& Oppenheimer, B. D. 2011, MNRAS, 416,1354

-. 2012, MNRAS, 421, 98

De Lucia, G., Kauffmann, G., \& White, S. D. M. 2004, MNRAS, 349,1101

Dekel, A., \& Silk, J. 1986, ApJ, 303, 39

Dickinson, M., Papovich, C., Ferguson, H. C., \& Budavári, T. 2003, ApJ, 587, 25

Erb, D. K. 2008, ApJ, 674, 151

Finlator, K., \& Davé, R. 2008, MNRAS, 385, 2181

Frenk, C. S., White, S. D. M., Davis, M., \& Efstathiou, G. 1988, ApJ, 327, 507

Gallazzi, A., Charlot, S., Brinchmann, J., White, S. D. M., \& Tremonti, C. A. 2005, MNRAS, 362, 41

Gnedin, N. Y. 2000, ApJ, 542, 535

Guo, Q., White, S., Boylan-Kolchin, M., et al. 2011, MNRAS, 413, 101

Henry, A., Martin, C. L., Finlator, K., \& Dressler, A. 2013a, ApJ, 769,148

Henry, A., Scarlata, C., Domínguez, A., et al. 2013b, ApJ, 776, L27

Houjun Mo, Frank van den Bosch, S. W. 2010, Galaxy Formation and Evolution, 1st edn. (Cambridge University Press)

Kacprzak, G. G., Martin, C. L., Bouché, N., et al. 2014, ApJ, 792, L12

Kewley, L. J., \& Ellison, S. L. 2008, ApJ, 681, 1183

Kirby, E. N., Cohen, J. G., Guhathakurta, P., et al. 2013, ApJ, 779,102

Kobayashi, C., Springel, V., \& White, S. D. M. 2007, MNRAS, 376,1465

Köppen, J., \& Edmunds, M. G. 1999, MNRAS, 306, 317

Kravtsov, A. V., Berlind, A. A., Wechsler, R. H., et al. 2004 , ApJ, 609, 35

Lacey, C., \& Silk, J. 1991, ApJ, 381, 14

Lee, H., Skillman, E. D., Cannon, J. M., et al. 2006, ApJ, 647, 970

Leitner, S. N. 2012, ApJ, 745, 149

Leitner, S. N., \& Kravtsov, A. V. 2011, ApJ, 734, 48

Leroy, A. K., Walter, F., Brinks, E., et al. 2008, AJ, 136, 2782

Lu, Y., Mo, H. J., Lu, Z., Katz, N., \& Weinberg, M. D. 2014, MNRAS, 443, 1252

Lu, Y., Mo, H. J., \& Wechsler, R. H. 2015a, MNRAS, 446, 1907

Lu, Y., Mo, H. J., Weinberg, M. D., \& Katz, N. 2011, MNRAS, 416,1949
Lu, Z., Mo, H. J., Lu, Y., et al. 2015b, MNRAS, 450, 1604

Ma, X., Hopkins, P. F., Faucher-Giguere, C.-A., et al. 2015 ArXiv e-prints, arXiv:1504.02097

Maiolino, R., Nagao, T., Grazian, A., et al. 2008, A\&A, 488, 463

Mannucci, F., Cresci, G., Maiolino, R., Marconi, A., \& Gnerucci, A. 2010, MNRAS, 408, 2115

Martin, C. L., Kobulnicky, H. A., \& Heckman, T. M. 2002, ApJ, 574,663

Matteucci, F., \& Tornambe, A. 1987, A\&A, 185, 51

McGaugh, S. S. 2005, ApJ, 632, 859

-. 2012, AJ, 143, 40

Melioli, C., Brighenti, F., \& D'Ercole, A. 2015, MNRAS, 446, 299

Moster, B. P., Naab, T., \& White, S. D. M. 2013, MNRAS, 428, 3121

Nagashima, M., Lacey, C. G., Baugh, C. M., Frenk, C. S., \& Cole, S. 2005, MNRAS, 358, 1247

Noh, Y., \& McQuinn, M. 2014, MNRAS, 444, 503

Nomoto, K., Hashimoto, M., Tsujimoto, T., et al. 1997a, Nuclear Physics A, 616, 79

Nomoto, K., Iwamoto, K., Nakasato, N., et al. 1997b, Nuclear Physics A, 621, 467

Papastergis, E., Cattaneo, A., Huang, S., Giovanelli, R., \&

Haynes, M. P. 2012, ApJ, 759, 138

Peeples, M. S., \& Somerville, R. S. 2013, MNRAS, 428, 1766

Peeples, M. S., Werk, J. K., Tumlinson, J., et al. 2014, ApJ, 786, 54

Pipino, A., Lilly, S. J., \& Carollo, C. M. 2014, MNRAS, 441, 1444

Searle, L., \& Sargent, W. L. W. 1972, ApJ, 173, 25

Somerville, R. S., Barden, M., Rix, H., et al. 2008, ApJ, 672, 776

Tinsley, B. M., \& Larson, R. B. 1978, ApJ, 221, 554

Tremonti, C. A., Heckman, T. M., Kauffmann, G., et al. 2004 ApJ, 613, 898

Weisz, D. R., Dolphin, A. E., Skillman, E. D., et al. 2014, ApJ, 789,147

White, S. D. M., \& Frenk, C. S. 1991, ApJ, 379, 52

Wiersma, R. P. C., Schaye, J., Theuns, T., Dalla Vecchia, C., \& Tornatore, L. 2009, MNRAS, 399, 574

Woosley, S. E., \& Heger, A. 2002, Reviews of Modern Physics, 74, 1015

Woosley, S. E., \& Weaver, T. A. 1995, ApJS, 101, 181

Yates, R. M., Henriques, B., Thomas, P. A., et al. 2013, MNRAS, 435,3500

Zahid, H. J., Dima, G. I., Kudritzki, R.-P., et al. 2014, ApJ, 791, 130

Zahid, H. J., Geller, M. J., Kewley, L. J., et al. 2013, ApJ, 771, L19 